\title{
Inhibition of platelet aggregation by olive oil phenols via cAMP-phosphodiesterase
}

\author{
Mario Dell'Agli*, Omar Maschi, Germana V. Galli, Rossana Fagnani, Esther Dal Cero, \\ Donatella Caruso and Enrica Bosisio \\ Research centre for the characterization and safe use of natural compounds- 'Giovanni Galli', \\ Department of Pharmacological Sciences, University of Milan, Italy
}

(Received 23 May 2007 - Revised 8 August 2007 - Accepted 24 August 2007 - First published online 11 October 2007)

The aim of the present study was to confirm that olive oil phenols reduce human platelet aggregability and to verify the hypothesis that cAMP- and cGMP- phosphodiesterases (PDE) could be one of the targets of the biological effect. Four extracts from oils characterized by a high phenol content (HPE), and low phenol levels (LPE) were prepared and analyzed quali- and quantitatively by HPLC-UV and electrospray ionization-MS/MS Human washed platelets stimulated with thrombin were used for the aggregation assay. Human platelet cAMP-PDE and recombinant PDE5A1 were used as enzyme source. Platelet aggregation and enzyme activity were assayed in the presence of HPE, LPE and individual phenols. The phenol content of HPE ranged between 250 and $500 \mathrm{mg} / \mathrm{kg}$, whereas the LPE content was $46 \mathrm{mg} / \mathrm{kg}$. The compounds identified were hydroxytyrosol (HT), tyrosol (TY), oleuropein aglycone (OleA) and the flavonoids quercetin (QU), luteolin (LU) and apigenin (AP). OleA was the most abundant phenol (range 23.3 to $37.7 \%$ ) and LU was the most abundant flavonoid in the extracts. Oil extracts inhibited platelet aggregation with an $50 \%$ inhibitory concentration interval of $1.23-11.2 \mu \mathrm{g} / \mathrm{ml}$. The inhibitory effect of individual compounds $(10 \mu \mathrm{M})$ including homovanillyl alcohol (HVA) followed this order: OleA $>\mathrm{LU}>\mathrm{HT}=\mathrm{TY}=\mathrm{QU}=\mathrm{HVA}$, while AP was inactive. All the extracts inhibited cAMP-PDE, while no significant inhibition of PDE5A1 $(50 \mu \mathrm{g} / \mathrm{ml})$ was observed. All the flavonoids and OleA inhibited cAMP-PDE, whereas HT, TY, HVA (100 $\mu \mathrm{M})$ were inactive. Olive oil extracts and part of its phenolic constituents inhibit platelet aggregation; cAMP-PDE inhibition is one mechanism through which olive oil phenols inhibit platelet aggregation.

Olive oil extracts: Platelet aggregation: Flavonoids: Phosphodiesterases: Phenols

A Mediterranean-style diet, where olive oil is the main source of fat, has been associated with a reduced risk of $\mathrm{CVD}^{1,2}$. Health benefits of olive oil have been in part attributed to minor phenol components, whose composition varies qualitatively and quantitatively depending on several factors such as the cultivar, stage of fruit ripeness and region of cultivation. In addition, the agronomic conditions and the process of oil extraction strongly affect the quality of olive oil and its content of phenols ${ }^{3}$.

The type of phenols in olive oil include flavonoids, in particular luteolin (LU) and apigenin (AP), and secoiridoids oleuropein aglycone (OleA), ligstroside aglycone and their hydrolysis products hydroxytyrosol (HT), and tyrosol (TY), respectively. Olive oil phenols possess antioxidant properties and influence many biological activities that may, at least partially, account for the observed effects of olive oil on the cardiovascular system. Some of these include: (1) inhibition of LDL oxidation $^{4-8},(2)$ production of nitric oxide ${ }^{9}$ and (3) the down regulation of intercellular adhesion molecule-1 and vascular cell adhesion molecule-1 expression in endothelial cells ${ }^{10,11}$ in the presence of olive oil phenols. Platelet aggregation, which often accompanies and aggravates CVD, was inhibited by olive oil phenols ${ }^{12}$, although this remains somewhat controversial ${ }^{13}$. Tight regulation of platelet function and platelet-vessel interaction is an essential requisite for intact vessel physiology. Platelet activation is regulated by a number of physiological activators (thromboxane $\mathrm{A}_{2}$, vasopressin, ADP, thrombin, serotonin) and inhibitors (endothelium-derived relaxing factor, prostaglandin inhibitor-2). Platelet antagonists inhibit platelet function by increasing the intracellular levels of cyclic nucleotides cAMP and cGMP through the activation of the respective cyclases. Cyclic nucleotide levels are down regulated by degradation through phosphodiesterases (PDE). Platelets contain mainly PDE3, which preferentially hydrolyzes cAMP as substrate, and PDE5 which uses preferentially cGMP as substrate ${ }^{14}$. PDE3 is inhibited by the binding of cGMP; therefore platelet PDE inhibition is reasonably considered a therapeutic tool to treat vascular diseases. Indeed PDE inhibitors are currently used as anti-aggregating agents and in the treatment of arterial occlusive diseases ${ }^{15}$.

Inhibition of platelet aggregation has been demonstrated for some olive oil phenols ${ }^{16}$. The exact nature of the mechanisms

Abbreviations: AP, apigenin; ESI, electrospray ionisation; HPE, high phenol extract; HT, hydroxytyrosol; HVA, homovanillyl alcohol; IC ${ }_{50}$, concentration that reduces the effect by $50 \%$; LPE, low phenol extract; LU, luteolin; OleA, oleuropein aglycone; PDE, phosphodiesterase; QU, quercetin; TY, tyrosol.

* Corresponding author: Dr Mario Dell'Agli, fax +39-0250318391, email mario.dellagli@unimi.it 
involved in the modulation of the platelet activity remains unclear. PDE inhibition has been speculated as one factor in this regard ${ }^{16}$, but there are no supporting data thus far.

In this paper we describe the effects of phenolic extracts obtained from five commercially available olive oils on aggregation of human platelets and on cAMP- and cGMP-PDE activities. The olive oils were chosen on the basis of their phenol content: four extracts (extracts A-D) were obtained from oils characterized by high phenol content (high phenol extract, HPE), while the fifth extract (extract E) was obtained from an olive oil with low phenol levels (low phenol extract, LPE). Each extract was analyzed qualitatively and quantitatively by HPLC-UV and electrospray ionization (ESI)-MS/MS in order to verify whether compositional differences affected biological activity.

\section{Experimental methods}

\section{Reagents}

Culture medium Dulbecco's modified Eagle's medium, trypsin, protease inhibitors, and all chemical reagents for cell culture were purchased from Sigma Aldrich (Milan, Italy). Penicillin, streptomycin, and L-glutamine were from GIBCO (Grand Island, NY, USA); foetal calf serum was provided by Mascia Brunelli S.p.A. (Milan, Italy). COS-7 cell line was purchased from ATCC (Manassas, VA, USA). Superfect reagent for transient transfections was obtained from Qiagen GmbH (Hilden, Germany). The expression plasmid pcDNA3 containing the full-length cDNA of PDE5A1 was a kind gift of Prof. C. S. Lin (Department of Urology, University of California, San Francisco, CA). $\left[{ }^{3} \mathrm{H}\right] \mathrm{cGMP}$ and $\left[{ }^{3} \mathrm{H}\right] \mathrm{cAMP}$ were from Amersham Pharmacia Biotech (Amersham Place, Little Chalfont, Buckinghamshire, UK). DEAESephadex A25 was from Pharmacia (Uppsala, Sweden). cGMP, cAMP, AMP, Crotalus adamanteus snake venom, quercetin (QU), aminophylline, phloretin, homovanillyl alcohol (HVA) and TY were purchased from Sigma Aldrich. Sildenafil was provided by Sequoia Research Products (Oxford, UK). AP and LU were purchased from Extrasynthèse (Lyon, France). OleA was obtained from oleuropein glucoside (Extrasynthèse, Lyon, France) by enzymatic digestion ${ }^{17}$, and the purity (99\%) was confirmed by both TLC and ESI-MS analysis. HT was from Cayman Chemical Company (Tallinn, Estonia). All compounds used for the analytical determinations and for the biological assays were of HPLC purity grade.

\section{Preparation and quantification of HPE and LPE total phenols}

Extracts A-E, obtained from olive oils available from various drugstores, were prepared according to the method of Montedoro et al. ${ }^{18}$ with minor modifications. Briefly, $100 \mathrm{~g}$ olive oil was delipidized with hexane $(100 \mathrm{ml})$ and extracted twice with methanol-water $(80: 20 \mathrm{v} / \mathrm{v} ; 100 \mathrm{ml})$ for $20 \mathrm{~min}$ on a mechanical shaker. The collected methanolic phases were then taken to dryness under $\mathrm{N}_{2}$ and the extracts stored at $-20^{\circ} \mathrm{C}$ until analysis. Spectrophotometric analyses of total phenols reactive to Folin-Ciocalteu ${ }^{19}$, expressed as oleuropein equivalents, were carried out as described ${ }^{20}$ (Fig. 1).

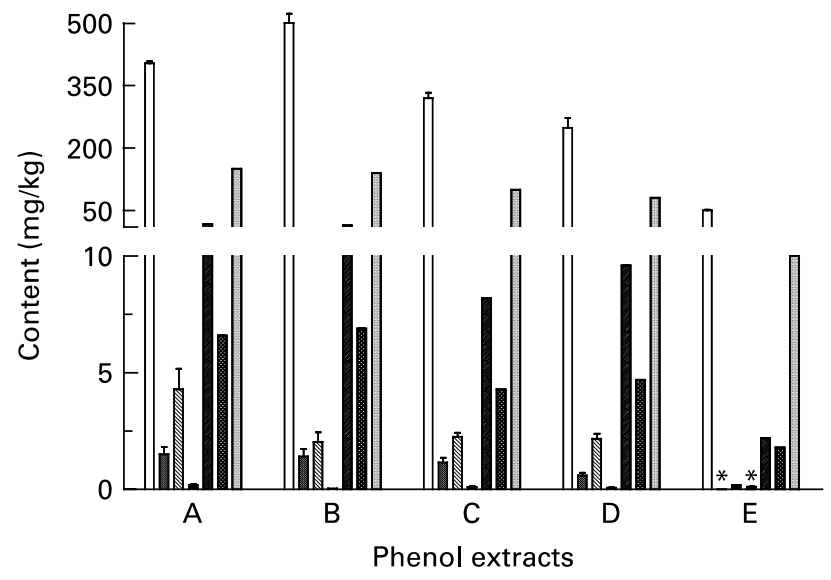

Fig. 1. Levels of phenols in olive oil extracts. A-D are extracts from oils with high phenol content (HPE), $E$ is from oil with low phenol content (LPE). Quantitative determination of the single phenols (apigenin (AP), 圆; luteolin

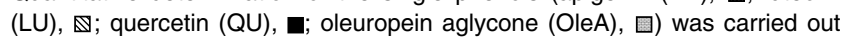
by electrospray ionization (ESI)-MS/MS equipped with an ESI source oper-

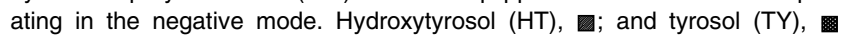
were quantified by GC-MS using deuterium-labelled compounds as internal standards. Results represent the mean and SD of at least three injections. Results were determined as phenols reactive to Folin-Ciocalteu, expressed as oleuropein equivalents. * Not detectable.

\section{Evaluation of HPE and LPE by HPLC-UV, GC-MS and ESI-} $M S / M S$

HPLC-UV analyses were performed on a JASCO instrument PU 980 using a LiChroCART ${ }^{\circledR} 4.0 \times 250 \mathrm{~mm} \quad(5 \mu \mathrm{m})$ HPLC-Cartridge Lichrosorb ${ }^{\circledR}$ RP-18 column (Merck, Darmstadt, Germany) at flow rate $1 \mathrm{ml} / \mathrm{min}$. The UV-VIS detector (Jasco mod 875-UV; Jasco Europe, Italy) was set at $278 \mathrm{~nm}$. A gradient elution was performed using water acidified with $2 \%$ acetic acid (A) and methanol (B). The gradient program was: $0-3 \mathrm{~min}, 90 \%$ (A); $3-13 \mathrm{~min}$, from $90 \%$ to $80 \%$ (A); $13-15 \mathrm{~min}, 80 \%$ (A); $15-20 \mathrm{~min}$, from $80 \%$ to $60 \%$ (A); $20-30 \mathrm{~min}$, from $60 \%$ to $50 \%(\mathrm{~A}) ; 30-50 \mathrm{~min}$, from $50 \%$ to $0 \%$ (A); $50-60 \mathrm{~min}, 0 \%$ (A); $60-65 \mathrm{~min}$, from $0 \%$ to $90 \%$ (A). Aliquots of HPE and LPE were dissolved in ethanol. OleA, HT, AP, QU, and LU were identified by comparison of the retention times and mass spectra with those of authentic standards. In the case of unresolved peaks, identification was obtained by co-injection of the extract with the authentic standards.

Quantitative determination of the single phenols was carried out by ESI-MS/MS using a linear ion-trap mass spectrometer (LTQ; Thermo Finnigan, USA) equipped with an ESI source operating in the negative mode. The operating parameters for $\mathrm{AP}, \mathrm{LU}$, and $\mathrm{QU}$ were as follows: source voltage $5 \mathrm{kV}$; source current $80 \mu \mathrm{A}$, capillary temperature and voltage were $250^{\circ} \mathrm{C}$ and $46 \mathrm{~V}$, respectively; sheath gas and auxiliary gas flow were 75 and 25 arbitrary units, respectively; tube lens offset $25 \mathrm{~V}$. For the quantification of OleA some modifications of the analytical parameters were used and in particular the capillary voltage was $35 \mathrm{~V}$, sheath gas and auxiliary gas flow were 80 and 5 arbitrary units, respectively and tube lens offset $15 \mathrm{~V}$. Collision energy was set at 50, 45 and $40 \%$ of $5 \mathrm{~V}$ for $\mathrm{AP}, \mathrm{LU}$, and $\mathrm{QU}$, respectively. All analyses were carried out by direct injection, through a loop injector. 
Calibration curves were prepared with phloretin (100 ng/ sample), as internal standard, and increasing amounts of the authentic phenols $(0-200 \mathrm{ng}$ for AP and QU, 0-500 ng for $\mathrm{LU})$; for the determination of OleA, samples for the calibration curve contained $0-100 \mu \mathrm{g}$ OleA and $100 \mu \mathrm{g}$ phloretin. Each sample was then extracted and analysed, as already described. Quantification was performed by monitoring ions derived from the collision of the $[\mathrm{M}-\mathrm{H}]^{-}$ion of each compound. One or more specific product ions were selected for each compound and used for quantitative analysis (Table 1).

HT and TY were quantified by GC-MS (GCQ, Thermoquest) using HT deuterium-labelled as internal standard ${ }^{21}$.

\section{Preparation of washed platelets for aggregation}

Venous blood from healthy volunteers was collected with sodium citrate $3.8 \%(9: 1 \mathrm{v} / \mathrm{v})$ and centrifuged at $160 \mathrm{~g}$ for $10 \mathrm{~min}$. The supernatant was centrifuged at $600 \mathrm{~g}$ for $6 \mathrm{~min}$ and the pellet solubilized in a buffer containing Tris- $\mathrm{HCl}$ $15.4 \mathrm{~mm}$, EDTA $2 \mathrm{~mm}$, glucose $5.5 \mathrm{~mm}, \mathrm{NaCl} 154 \mathrm{~mm}, \mathrm{pH}$ 7.4. After centrifugation at $600 \mathrm{~g}$ for $20 \mathrm{~min}$, pellet was solubilized in Tyrode-HEPES buffer $(\mathrm{NaCl} 137 \mathrm{mM}, \mathrm{KCl}$ $2.68 \mathrm{~mm}, \mathrm{NaHCO}_{3} 11.9 \mathrm{~mm}, \mathrm{NaH}_{2} \mathrm{PO}_{4} 0.416 \mathrm{~mm}, \mathrm{MgCl}_{2}$ $1 \mathrm{~mm}$, destrose $0.55 \mathrm{~mm}$, HEPES $5 \mathrm{~mm}, \mathrm{pH} 7.35$ ). Platelet number was adjusted to $1 \times 10^{8}$ platelets $/ \mathrm{ml}$ with buffer. All the procedure was performed at room temperature.

Aggregation was carried out in an Elvi 840 aggregometer (Elvi Logos, Milano, Italy). Washed platelets $(250 \mu \mathrm{l})$ were pre-incubated at $37^{\circ} \mathrm{C}$, stirring at $1000 \mathrm{rpm}$ for $10 \mathrm{~min}$ in the presence or in the absence of the compounds to be tested, and stimulated with thrombin $1 \mathrm{U} / \mathrm{ml}$ (Sigma, Milano, Italy). The reaction lasted $5 \mathrm{~min}$. Oil extracts were tested in the concentration range of $0 \cdot 1-20 \mu \mathrm{g} / \mathrm{ml}$, and single compounds were tested at $10 \mu \mathrm{M}$. The concentration of the vehicle in the control and treated samples was $0 \cdot 1 \%$.

\section{Platelet homogenate preparation and assay for cAMP-PDE} activity

The blood fraction enriched in platelets, obtained from blood of healthy volunteers, was submitted to two centrifugations at $160 \mathrm{~g}$ for $10 \mathrm{~min}$ at room temperature. The pellet was removed and platelet-rich plasma was centrifuged at $1000 \mathrm{~g}$ for $15 \mathrm{~min}$. The resulting pellet was resuspended in $10 \mathrm{~mm}$ Tris- $\mathrm{HCl}, \mathrm{pH}$ 7.4 (2/5 of the initial volume). The suspension was centrifuged

Table 1. Precursor and product ions selected for ESI-MS/MS analysis. Quantification was performed by monitoring ions derived from the collision of the $[\mathrm{M}-\mathrm{H}]^{-}$ion of each compound. One or more specific product ions were selected for each compound and used for quantitative analysis

\begin{tabular}{lcr}
\hline Compound & Precursor ion $(\mathrm{m} / \mathrm{z})[\mathrm{M}-\mathrm{H}]^{-}$ & \multicolumn{1}{c}{ Product ion $(\mathrm{m} / \mathrm{z})$} \\
\hline OleA & 377 & 307 \\
AP & 269 & $148-150,182,201,225$ \\
LU & 285 & $151,175,199,241,285$ \\
QU & 301 & 179 \\
Phloretin & 273 & 167
\end{tabular}

OleA, oleuropein aglycone; AP, apigenin; LU, luteolin; QU, quercetin. at $1000 \mathrm{~g}$ for $15 \mathrm{~min}$ and the pellet suspended in the Tris- $\mathrm{HCl}$ buffer, $\mathrm{pH} 7.4$ (1/12 of the initial volume). All these steps were performed at $4^{\circ} \mathrm{C}$. Cells were disrupted by freezing and thawing three times obtaining the homogenate ${ }^{22}$, and cell lysates was stored at $-80^{\circ} \mathrm{C}$. Total protein concentration was measured according to Bradford ${ }^{23}$.

cAMP-PDE activity was determined according to the method of Kincaid \& Manganiello ${ }^{24}$ with minor modifications. Platelet lysate $(64 \mu \mathrm{g}$ protein $/ \mathrm{ml})$ was incubated with $0.5 \mu \mathrm{M}$-cAMP and $63 \mathrm{nCi}\left[{ }^{3} \mathrm{H}\right] \mathrm{cAMP}$ suspended in $30 \mathrm{mM}$ Tris- $\mathrm{HCl}, \mathrm{pH} 7.4,4 \mathrm{mM} \mathrm{MgCl}_{2}$; final reaction volume was $250 \mu \mathrm{l}$. After $5 \mathrm{~min}$ incubation at $30^{\circ} \mathrm{C}$, the reaction was stopped with $0 \cdot 1 \mathrm{M} \mathrm{HCl}$. Samples were then incubated for a further $4 \mathrm{~min}$ at $70^{\circ} \mathrm{C}$ with AMP $5 \mathrm{mM}$ and cAMP $5 \mathrm{mM}$, and the $\mathrm{pH}$ adjusted to 7 on ice with $0.1 \mathrm{M} \mathrm{NaOH}$. Samples were then incubated for $20 \mathrm{~min}$ at $37^{\circ} \mathrm{C}$ with $50 \mu \mathrm{l}$ nucleotidase from Crotalus adamanteus snake venom $(1 \mathrm{mg} / \mathrm{ml}$ in Tris-HCl 0.1 M, pH 8.0) to cleave AMP to the corresponding nucleoside. The reaction was stopped with $50 \mu \mathrm{l} 200 \mathrm{~mm}$ NaEDTA containing $5 \mathrm{~mm}$ adenosine. The nucleoside formed during the incubation was separated from the unreacted substrate by dietylamino ethanol-Sephadex A25 column chromatography. The eluted $\left[{ }^{3} \mathrm{H}\right]$ adenosine was counted in a $\beta$-scintillation counter. cAMP-PDE activity was expressed as picomoles of product formed per minute per milligram of protein. HPE and LPE were tested at $1-100 \mu \mathrm{g} / \mathrm{ml}$, while concentration-response curves of the single compounds ranged between $0 \cdot 1$ and $25 \mu \mathrm{M}$. Concentrations reducing the effect by $50 \%\left(\mathrm{IC}_{50}\right)$ were calculated from the sigmoydal curves using Graph Pad Prism 4. Inhibition (\%) by aminophylline $100 \mu \mathrm{M}$ used as reference compound was 74.5 (SD $1.30 ; n 11)$. Each result is the mean and standard deviation of two experiments in triplicate.

Expression and enzyme assay of human recombinant PDE5Al

Human recombinant PDE5A1 was prepared by expression of the full-length cDNA of PDE5A1 into COS-7 cells as previously described ${ }^{25}$. PDE5A1 activity was determined according to the method of Kincaid \& Manganiello ${ }^{24}$ with minor modifications ${ }^{26}$. The extracts were assayed at $50 \mu \mathrm{g} / \mathrm{ml}$, expressed as total phenol content, whereas the pure compounds were tested at $50 \mu \mathrm{M}$. The PDE-5 inhibitor sildenafil used as reference compound showed $70 \%$ inhibition at $100 \mathrm{nM}$.

\section{Results}

\section{Phenol content of HPE and LPE}

The total phenol content of HPE ranged between 250 and $500 \mathrm{mg} / \mathrm{kg}$ as expected, whereas the LPE content was $46 \mathrm{mg} / \mathrm{kg}$ (Fig. 1). The compounds identified were the phenolic alcohols HT and TY, OleA and the flavonoids QU, LU, and AP. LPE showed a qualitatively, but not quantitatively, similar profile. The results of the quantitative determination of the identified compounds are shown in Fig. 1. OleA was the most abundant phenol present in the extracts (range 23.3 to $37.7 \%$ ) and LU was the most abundant flavonoid in all the extracts analyzed. LPE showed the lowest amount of OleA and LU, while AP and QU were undetectable. 
Effect of oil extracts and single phenols on platelet aggregation and PDE activities

In order to determine whether oil phenols inhibit platelet aggregation, human washed platelets were first stimulated with thrombin in the presence of oil extracts. Under these conditions the oil extracts inhibited platelet aggregation in a concentration-dependent fashion (Fig. 2), with an $\mathrm{IC}_{50}$ interval of $1.23-11.2 \mu \mathrm{g} / \mathrm{ml}$, (Table 2). When the individual compounds were tested similarly, the inhibitory effect obtained at $10 \mu \mathrm{M}$ followed this order: OleA $>\mathrm{LU}>\mathrm{HT}=\mathrm{TY}=\mathrm{QU}=\mathrm{HVA}$, while AP was inactive.

Since it is known that reduced platelet aggregation is associated with elevated levels of cAMP, the next step was to verify the ability of oil phenols to inhibit cAMP-PDE and cGMP-PDE-5. All the extracts inhibited cAMP-PDE in human platelet lysates, again in a concentration-dependent manner (Fig. 3). The observed $\mathrm{IC}_{50}$ in this instance ranged from 14.2 to $33.8 \mu \mathrm{g} / \mathrm{ml}$ for $\mathrm{HPE}$, and was 28.2 (SD 2.5) $\mu \mathrm{g} / \mathrm{ml}$ for LPE (Table 2). On the other hand, no significant inhibition of PDE5A1 occurred at concentration as high as $50 \mu \mathrm{g} / \mathrm{ml}$ (data not shown).

All individual compounds were tested on cAMP-PDE, as well. HVA, although absent in oil extracts, was included in these studies since it is the main HT metabolite in human subjects ${ }^{27,28}$. All the flavonoids showed a concentration-dependent inhibition of cAMP-PDE (Fig. 4). The $\mathrm{IC}_{50}$ are reported in Table 2. Among the non-flavonoidic constituents tested, only OleA showed a concentration-dependent inhibition, although at higher concentrations (Fig. 4, Table 2), whereas HT, TY, and HVA were inactive up to $100 \mu \mathrm{M}$.

In order to verify whether the lack of inhibition of PDE5A1 by oil extracts could be due to the inability of the identified constituents to inhibit the enzyme, PDE5A1 was assayed in the presence of AP, LU, QU, OleA, HT and TY at $50 \mu \mathrm{M}$. The inhibition by AP, LU and QU of PDE5A1 was 21.5 (SD 5.7), 50.9 (SD 2.2) and 44.1 (SD $8 \cdot 1) \%$, respectively lower in comparison with the inhibition

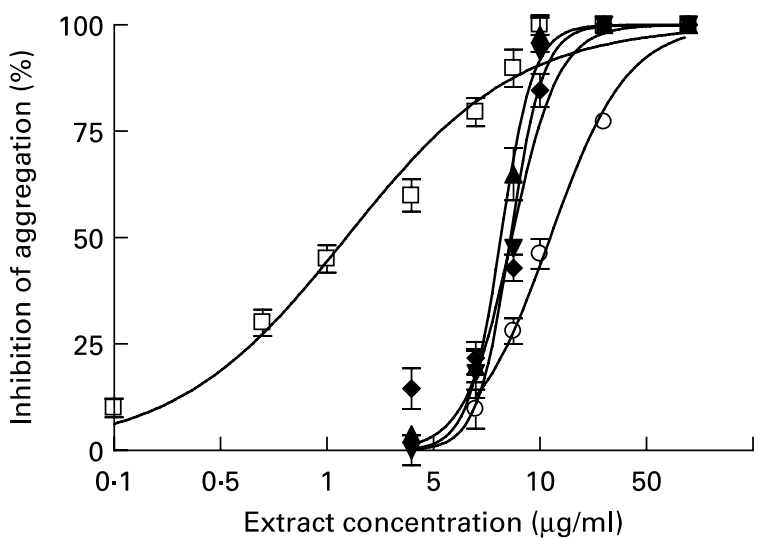

Fig. 2. Effect of high phenol extract (HPE) oils $(A, \square ; B, \mathbf{\Lambda} ; C, \mathbf{v}$; and $D, \bullet)$ and low phenol extract (LPE; $E, O)$ on human platelet aggregation. Human washed platelets $(250 \mu \mathrm{l})$ were pre-incubated at $37^{\circ} \mathrm{C}$, stirring at $1000 \mathrm{rpm}$ for $10 \mathrm{~min}$ in the presence or in the absence of the extracts $(1-20 \mu \mathrm{g} / \mathrm{ml})$, and stimulated with thrombin $1 \mathrm{U} / \mathrm{ml}$. Each result is the mean of three experiments with standard deviation indicated by vertical bars. of cAMP-PDE. OleA had a rather moderate effect: 9.3 (SD 2.7) $\%$ inhibition at $50 \mu \mathrm{M}$ and TY was inactive. While HT inhibited PDE5A1 activity in a concentrationdependent fashion $\left(\mathrm{IC}_{50} 40 \cdot 6\right.$ (SD 7.3) $\left.\mu \mathrm{M}\right)$, it was without effect on cAMP-PDE.

\section{Discussion}

In the present study our objective was to confirm that various olive oil phenols reduce human platelet aggregability and to verify the hypothesis that the cAMP and cGMP dependent PDE could be one of the targets of the biological effect. For this purpose five commercially available olive oils were processed to obtain extracts with differing phenol contents, both qualitatively and quantitatively. The phenol content in four oils (HPE) ranged between 250 and $500 \mathrm{mg} / \mathrm{kg}$ in agreement with the previously reported data ${ }^{29,30}$. In LPE, phenol content was from 5- to 10-fold lower than that of HPE; most likely due to the chemical treatment required to reduce the exceedingly high acidity of this olive oil. HPLC-UV analysis of the extracts showed a complex profile and some peaks could be clearly identified as HT, TY, OleA, $\mathrm{AP}, \mathrm{QU}$, and LU, and quantified. Cultivar, ripening stage, and production technologies (used to obtain olive oils) could explain the observed levels and compositional differences.

This report shows that olive oil extracts and part of their phenolic constituents inhibit platelet aggregation. Our results disagree with those obtained by Turner et al. ${ }^{13}$, which reported no effect by olive oil phenols on collagen-challenged platelet in blood, thus in assay conditions differing from ours. The concentration of the identified compounds in oil extracts, and the extent to which they inhibit platelet aggregation are not consistent with the inhibitory activity shown by the whole extracts. It is thus likely that HPE contain unidentified constituents with anti-aggregating activity. These could include isochromans, a new class of compounds recently described to occur in olive oil which were shown to inhibit human platelet reactivity ${ }^{31}$

According to Petroni et $\mathrm{al}^{12}{ }^{12}$, inhibition of platelet aggregation is thought to be mediated by a decrease in eicosanoid production caused by phenols. In addition to affecting the arachidonic acid cascade, our data show that the mechanism of action could also be through a degradation of cAMP brought about by phenols, since HPE and LPE inhibited cAMP-PDE. Conversely cGMP-PDE5 seems to be less or not affected at all by olive oil phenols when tested as a mixture. AP, LU and QU, OleA and HT, also inhibited PDE5A1, however their concentration in the phenol mixture was insufficient to cause enzyme inhibition. QU, AP, LU and OleA contributed differently to the overall effect of the extract on cAMP-PDE, while HT and TY, the in vivo metabolites of OleA and ligstroside aglycone respectively, were inactive. Taking into consideration the level of each compound (as \% of the total phenols) and the $\mathrm{IC}_{50}$ of each phenol, LU would be the major component to inhibit platelet aggregation among the identified compounds. Other components of olive oil, still of unknown nature, could play a role in this process as well. A possible explanation for the lower inhibitory activity of HPE-D is the likely presence of compounds that may counteract the 
Table 2. Effects of olive oil extracts and single phenols on platelet aggregation and cAMP-PDE activity. Individual compounds were tested on platelet aggregation at $10 \mu \mathrm{M}$

(Mean values and standard deviations)

\begin{tabular}{|c|c|c|c|c|}
\hline \multirow[b]{2}{*}{ Compound } & \multicolumn{2}{|c|}{ Platelet aggregation $\mathrm{IC}_{50}(\mu \mathrm{g} / \mathrm{ml})$} & \multicolumn{2}{|c|}{ cAMP-PDE IC $50(\mu \mathrm{g} / \mathrm{ml})$} \\
\hline & Mean & SD & Mean & SD \\
\hline \multicolumn{5}{|c|}{ Olive oil extract } \\
\hline HPE-A & 1.23 & 0.24 & $14 \cdot 2$ & 0.3 \\
\hline HPE-B & 6.54 & 0.23 & $15 \cdot 4$ & 0.4 \\
\hline HPE-C & 6.83 & 0.53 & $15 \cdot 9$ & 0.8 \\
\hline HPE-D & 7.38 & 0.69 & $33 \cdot 8^{*}$ & $2 \cdot 7$ \\
\hline LPE & $11 \cdot 17$ & 0.68 & $28 \cdot 2^{*}$ & $2 \cdot 5$ \\
\hline & \multicolumn{2}{|c|}{ Inhibition (\%) } & \multicolumn{2}{|c|}{$\mathrm{IC}_{50}(\mu \mathrm{M})$} \\
\hline Phenol & & & & \\
\hline OleA & $75 \cdot 4$ & 4.5 & $89 \cdot 0$ & $4 \cdot 3$ \\
\hline LU & $23 \cdot 3$ & 0.7 & 1.3 & 0.1 \\
\hline $\mathrm{AP}$ & \multicolumn{2}{|c|}{ Inactive } & $4 \cdot 1$ & 0.9 \\
\hline QU & 7.5 & $2 \cdot 9$ & 1.5 & 0.3 \\
\hline HT & 11.4 & 3.4 & \multicolumn{2}{|c|}{ Inactive at $100 \mu \mathrm{M}$} \\
\hline TY & $10 \cdot 2$ & 2.5 & \multicolumn{2}{|c|}{ Inactive at $100 \mu \mathrm{M}$} \\
\hline HVA & 6.8 & 4.4 & \multicolumn{2}{|c|}{ Inactive at $100 \mu \mathrm{M}$} \\
\hline
\end{tabular}

PDE, phophodiesterase; HPE, high phenol extract; LPE, low phenol extract; OleA, oleuropein aglycone; LU, luteolin; AP, apigenin; QU, quercetin; HT, hydroxytyrosol; TY, tyrosol; HVA, homovanillyl alcohol.

* Significantly different $v$. A-C.

effects of the active principles. The activity of LPE is lower with respect to HPE $\mathrm{A}-\mathrm{C}$, but the degree of inhibition is consistent with the low levels of LU and OleA, and with the absence of AP. Thus the composition and the quantity of phenols in oil extracts affect the degree of inhibition of cAMP-PDE.

The in vivo significance of the results presented here with regard to inhibition of platelet aggregation by olive oil remains to be established. Quite limited data are available on the bioavailability of $\mathrm{HT}$ and $\mathrm{TY}^{30,32}$, and only one study $^{33}$ addressed the question of evaluating the urinary levels of these two compounds after olive oil intake. According to those studies OleA, HT, and TY are adsorbed, extensively modified in the body, and HT and TY are present in plasma only in the conjugated forms ${ }^{28}$. The maximum

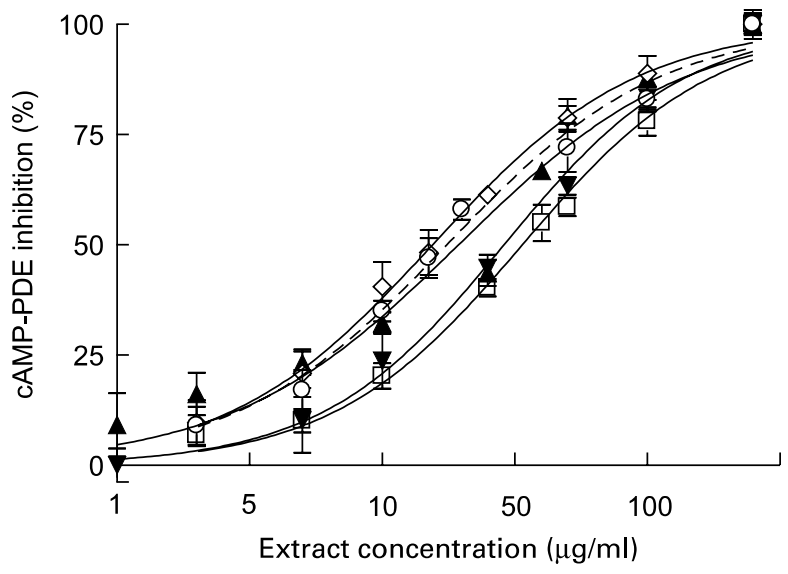

Fig. 3. Effect of high phenol extract (HPE: A, $\mathbf{\Delta} ; \mathrm{B}, \diamond ; \mathrm{C}, \mathrm{O}$; and $\mathrm{D}, \square$ ) and low phenol extract (LPE; E, $\mathbf{\nabla}$ ) on human platelet cAMP-phosphodiesterase (CAMP-PDE). HPE and LPE were tested at $1-100 \mu \mathrm{g} / \mathrm{ml}$. CAMP-PDE activity was determined according to the method of Kincaid \& Manganiello ${ }^{24}$ with minor modifications. Results are the means of two experiments performed in triplicate with standard deviation indicated by vertical bars. concentration of flavonoids in human plasma rarely exceeds $1 \mu \mathrm{M}^{34}$ but bioavailability studies on flavonoids were performed after intake of non-oil matrices. When HT was administered as a natural component of olive oil, its urinary excretion in human subjects was higher than after its addition to refined olive oil or yoghurt ${ }^{35}$. Similarly flavonoids might be more bio-available after olive oil intake.

Finally, since conjugated HT could not be assessed for platelet aggregation due to its current unavailability, the effect of olive oil phenols should be attributed to components of stillunknown structure.

Consumption of olive oil has long been considered to be healthy, particularly with regard to its effects on the cardiovascular system. The role of platelets is increasingly well defined in coronary artery disease and platelet aggregation

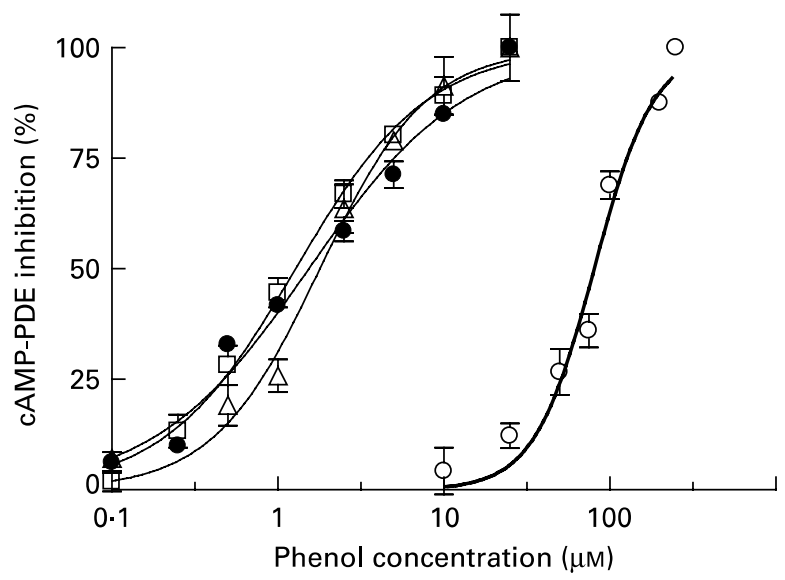

Fig. 4. Effect of luteolin (LU, $\square$ ), apigenin (AP, $\Delta$ ), quercetin $(\mathrm{QU}, \mathbf{\bullet})$, and oleuropein aglycone $(\mathrm{OleA}, \mathrm{O})$ on human platelet cAMP-phosphodiesterase (CAMP-PDE). Concentration-response curves of the single compounds ranged between 0.1 and $25 \mu \mathrm{M}$. cAMP-PDE activity was determined according to the method of Kincaid \& Manganiello ${ }^{24}$. Results are the means of two experiments performed in triplicate with standard deviation indicated by vertical bars. 
is shown to be one underlying mechanism in its pathogenesis. Phenolic components of commonly consumed foods, in Mediterranean countries at least, such as red wine and olive oil rich in these constituents, are shown to inhibit platelet aggregation thus potentially reducing the putative risk of vascular diseases. Data presented here show one mechanism, cAMP-PDE inhibition, by which olive oil phenols inhibit platelet aggregation.

\section{Acknowledgements}

Prof. C. S. Lin, Department of Urology, University of California, San Francisco, CA, USA, for the kind supply of PDE5A1 cDNA, and Mrs M. P. Pasini for generous financial support are gratefully acknowledged. The authors thank Prof. C. Galli for the access to the aggregometer, Dr S. Barbieri for the help in the aggregation assay, and $\mathrm{Mr}$ A. Toia for technical assistance. The authors thank Dr A. Sanghvi for valuable comments.

\section{References}

1. Estruch R, Martinez-Gonzalez MA, Corella D, et al. (2006) Effects of a Mediterranean-style diet on cardiovascular risk factors: a randomized trial. Ann Intern Med 145, 1-11.

2. Serra-Majem L, Roman B \& Estruch R (2006) Scientific evidence of interventions using the Mediterranean diet: a systematic review. Nutr Rev 64, S27-S47.

3. Servili M \& Montedoro G (2002) Contribution of phenolic compounds to virgin olive oil quality. Eur J Lipid Sci Tech 104, 602-613.

4. Visioli F, Bellomo G, Montedoro G \& Galli C (1995) Low density lipoprotein oxidation is inhibited in vitro by olive oil constituents. Atherosclerosis 117, 25-32.

5. Caruso D, Berra B, Giavarini F, Cortesi N, Fedeli E \& Galli G (1999) Effect of virgin olive oil phenolic compounds on in vitro oxidation of human low density lipoproteins. Nutr Metab Cardiovasc Dis 9, 102-107.

6. Andrikopoulos NK, Kaliora AC, Assimopoulou AN \& Papageorgiou VP (2002) Inhibitory activity of minor polyphenolic and non polyphenolic constituents of olive oil against in vitro low-density lipoprotein oxidation. J Med Food 5, 1-7.

7. Benkhalti F, Legssyer A, Gomez P, Paz E, Lopez-Miranda J, Perez-Jimenez F \& el Boustani ES (2003) Effects of virgin olive oil phenolic compounds on LDL oxidation and vasorelaxation activity. Therapie 58, 133-137.

8. Ferroni F, Maccaglia A, Pietraforte D, Turco L \& Minetti M (2004) Phenolic antioxidants and the protection of low density lipoprotein from peroxynitrite-mediated oxidations at physiologic $\mathrm{CO}_{2}$. J Agric Food Chem 52, 2866-2874.

9. Visioli F, Bellosta S \& Galli C (1998) Oleuropein, the bitter principle of olives, enhances nitric oxide production by mouse macrophages. Life Sci 62, 541-546.

10. Carluccio MA, Siculella L, Ancora MA, Massaro M, Scoditti E, Storelli C, Visioli F, Distante A \& De Caterina R (2003) Olive oil and red wine antioxidant polyphenols inhibit endothelial activation: antiatherogenic properties of Mediterranean diet phytochemicals. Arterioscler Thromb Vasc Biol 23, 622-629.

11. Dell'Agli M, Fagnani R, Mitro N, et al. (2006) Minor components of olive oil modulate proatherogenic adhesion molecules involved in endothelial activation. J Agric Food Chem 54, 3259-3264.

12. Petroni A, Blasevich M, Salami M, Papini N, Montedoro GF \& Galli C (1995) Inhibition of platelet aggregation and eicosanoid production by phenolic components of olive oil. Thromb Res $\mathbf{7 8}$, $151-160$

13. Turner R, Etienne N, Alonso MG, de Pascual-Teresa S, Minihane AM, Weinberg PD \& Rimbach G (2005) Antioxidant and anti-atherogenic activities of olive oil phenolics. Int $J$ Vitam Nutr Res 75, 61-70.

14. Haslam RJ, Dickinson NT \& Jang EK (1999) Cyclic nucleotides and phosphodiesterases in platelets. Thromb Haemost 82, 412-423.

15. Bender AT \& Beavo JA (2006) Cyclic nucleotide phosphodiesterases: molecular regulation to clinical use. Pharmacol Rev 58, $488-520$

16. Natella F, Nardini M, Virgili F \& Scaccini C (2006) Role of dietary polyphenols in the platelet aggregation network a review of the in vitro studies. Curr Topics Nutr Res 4, $1-21$.

17. Limiroli RC, Ottolina G, Marsilio V, Bianchi G \& Zetta L (1995) ${ }^{13} \mathrm{C}$ NMR characterisation of new oleuropein aglycons. J Chem Soc Perkin Trans 1, 1519-1523.

18. Montedoro GS, Baldioli M \& Miniati E (1992) Simple and hydrolizable phenolic compounds in virgin olive oil. 1. Their extraction, separation, quantitative and semiquantitative evaluation by HPLC. J Agric Food Chem 40, 1571-1576.

19. Folin O \& Ciocalteu V (1927) On tyrosine and tryptophane determinations in proteins. J Biol Chem 73, 627-650.

20. Cardoso SM, Guyot S, Marnet N, Lopes-da-Silva JA, Renard CMGC \& Coimbra MA (2005) Characterization of phenolic extracts from olive pulp and olive pomace by electrospray mass spectrometry. J Sci Food Agric 85, 21-32.

21. Visioli F, Caruso D, Galli C, Viappiani S, Galli G \& Sala A (2000) Olive oils rich in natural catecholic phenols decrease isoprostane excretion in humans. Biochem Biophys Res Commun 278, 797-799.

22. Giovanazzi S, Accomazzo MR, Letari O, Oliva D \& Nicosia S (1997) Internalization and down-regulation of the prostacyclin receptor in human platelets. Biochem J 325, 71-77.

23. Bradford MM (1976) A rapid and sensitive method for the quantitation of microgram quantities of protein utilizing the principle of protein-dye binding. Anal Biochem 72, $248-254$.

24. Kincaid RL \& Manganiello VC (1988) Assay of cyclic nucleotide phosphodiesterase using radiolabeled and fluorescent substrates. Methods Enzymol 159, 457-470.

25. Lin CS, Lau A, Tu R \& Lue TF (2000) Expression of three isoforms of cGMP-binding cGMP-specific phosphodiesterase (PDE5) in human penile cavernosum. Biochem Biophys Res Commun 268, 628-635.

26. Dell'Agli M, Galli GV, Vrhovsek U, Mattivi F \& Bosisio E (2005) In vitro inhibition of human cGMP-specific phosphodiesterase- 5 by polyphenols from red grapes. J Agric Food Chem 53, 1960-1965.

27. Visioli F, Galli C, Bornet F, Mattei A, Patelli R, Galli G \& Caruso D (2000) Olive oil phenolics are dose-dependently absorbed in humans. FEBS Lett 468, 159-160.

28. Miro-Casas E, Covas MI, Farre M, Fito M, Ortuno J, Weinbrenner T, Roset P \& de la Torre R (2003) Hydroxytyrosol disposition in humans. Clin Chem 49, 945-952.

29. Visioli F \& Galli C (2002) Biological properties of olive oil phytochemicals. Crit Rev Food Sci Nutr 42, 209-221.

30. Vissers MN, Zock PL \& Katan MB (2004) Bioavailability and antioxidant effects of olive oil phenols in humans: a review. Eur $J$ Clin Nutr 58, 955-965.

31. Togna GI, Togna AR, Franconi M, Marra C \& Guiso M (2003) Olive oil isochromans inhibit human platelet reactivity. $J$ Nutr 133, 2532-2536.

32. Bonanome A, Pagnan A, Caruso D, Toia A, Xamin A, Fedeli E, Berra B, Zamburlini A, Ursini F \& Galli G (2000) Evidence of 
postprandial absorption of olive oil phenols in humans. Nutr Metab Cardiovasc Dis 10, 111-120.

33. Miro-Casas E, Covas MI, Fito M, Farre-Albadalejo M, Marrugat J \& de la Torre R (2003) Tyrosol and hydroxytyrosol are absorbed from moderate and sustained doses of virgin olive oil in humans. Eur J Clin Nutr 57, 186-190.
34. Scalbert A \& Williamson G (2000) Dietary intake and bioavailability of polyphenols. J Nutr 130, S2073-S2085.

35. Visioli F, Galli C, Grande S, Colonnelli K, Patelli C, Galli G \& Caruso D (2003) Hydroxytyrosol excretion differs between rats and humans and depends on the vehicle of administration. $J$ Nutr 133, 2612-2615. 